\title{
Stimulation of TRPV1 by Green Laser Light
}

\author{
Quanbao Gu, ${ }^{1}$ Lina Wang, ${ }^{1,2}$ Fang Huang, ${ }^{3}$ and Wolfgang Schwarz ${ }^{1,4}$ \\ ${ }^{1}$ Shanghai Research Center for Acupuncture and Meridians, 199 Guoshoujing Road, Shanghai 201203, China \\ ${ }^{2}$ Acupuncture and Moxibution College, Shanghai University of Traditional Chinese Medicine, 1200 Cailun Road, \\ Shanghai 201203, China \\ ${ }^{3}$ State Key Laboratory of Medical Neurobiology, Shanghai Medical College, Fudan University, Shanghai 200031, China \\ ${ }^{4}$ Institute for Biophysics, J. W. Goethe University, Max-von-Laue Straße 1, 60438 Frankfurt am Main, Germany
}

Correspondence should be addressed to Wolfgang Schwarz, schwarz@biophys.eu

Received 20 September 2012; Revised 6 November 2012; Accepted 14 November 2012

Academic Editor: Di Zhang

Copyright (C) 2012 Quanbao Gu et al. This is an open access article distributed under the Creative Commons Attribution License, which permits unrestricted use, distribution, and reproduction in any medium, provided the original work is properly cited.

\begin{abstract}
Low-level laser irradiation of visible light had been introduced as a medical treatment already more than 40 years ago, but its medical application still remains controversial. Laser stimulation of acupuncture points has also been introduced, and mast-cells degranulation has been suggested. Activation of TRPV ion channels may be involved in the degranulation. Here, we investigated whether TRPV1 could serve as candidate for laser-induced mast cell activation. Activation of TRPV1 by capsaicin resulted in degranulation. To investigate the effect of laser irradiation on TRPV1, we used the Xenopus oocyte as expression and model system. We show that TRPV1 can functionally be expressed in the oocyte by (a) activation by capsaicin $\left(K_{1 / 2}=1.1 \mu \mathrm{M}\right)$, (b) activation by temperatures exceeding $42^{\circ} \mathrm{C}$, (c) activation by reduced $\mathrm{pH}$ (from 7.4 to 6.2), and (d) inhibition by ruthenium red. Red (637 nm) as well as blue $(406 \mathrm{~nm})$ light neither affected membrane currents in oocytes nor did it modulate capsaicin-induced current. In contrast, green laser light ( $532 \mathrm{~nm}$ ) produced power-dependent activation of TRPV1. In conclusion, we could show that green light is effective at the cellular level to activate TRPV1. To which extend green light is of medical relevance needs further investigation.
\end{abstract}

\section{Introduction}

Low-level laser irradiation in the $\mathrm{mW} / \mathrm{cm}^{2}$ range of visible and near-infrared (NIR) light had been introduced as a medical treatment already in the late 60 s (see [1]), but its medical application still remains controversial. One major reason is that the cellular and molecular photo/biological responses are highly complex.

Since light below $600 \mathrm{~nm}$ is strongly absorbed in tissue by haemoglobin and melanin, and above $1200 \mathrm{~nm}$ by the water, medical application focused on the red and NIR range (see e.g., [2]). As possible photoreceptor cytochrome oxidase $\mathrm{C}$ has been suggested $[3,4]$. Also in a modern variant of Chinese medicine, laser light is used to stimulate acupuncture points (see e.g., [5]). In traditional Chinese medicine, acupuncture points are stimulated by the needling procedure; it could be demonstrated that this leads to the degranulation of mast cells, which forms an essential early step in acupuncture-induced analgesia [6]. The degranulation in acupuncture points cannot only be induced by the mechanical stress during needle manipulation or osmotic stress, but also by high temperatures like those that are applied during moxibustion. Even irradiation of mast cells with red laser light, that is used in the laser acupuncture $[5,7]$, results in degranulation [8]. More recently, irradiation of acupoints with blue laser light has been introduced in medical treatment [9].

In the work by Zhang et al. [8], it was shown that the degranulation induced by mechanical stress, high temperature, and red light involves activation of TRPV2, a member of the family of transient-receptor-potential (TRP) ion channels. Application of blue laser light was also demonstrated to elicit mast-cell degranulation, and the involvement of another TRP channel, TRPV4, was suggested [10]. In addition to these two TRPV channels, mast cells also express TRPV1 [8]. In our work presented here we investigated to which extent TRPV1 can be activated by blue, green, and red laser light.

To investigate effects of laser light on TRPV1 and to avoid interference with the other members of TRPV family in the 
mast cells, we used Xenopus oocytes as a model system with heterologously expressed TRPV1. Activity of TRPV1 was monitored under voltage clamp as TRPV1-mediated current.

\section{Materials and Methods}

2.1. Transcription of TRPV1 cDNA. Full length human TRPV1 cDNA was cut from the vector pCAGGSM2-IRES-GFPR1R2/TrpV1 (kindly provided by Dr. B. Nilius, University of Leuven, Belgium) with restriction enzyme Cla I and EcoR I and subcloned into the in vitro transcription vector pTLN digested with the same enzymes. The recombinant plasmid named pTLN-hTRPV1 was confirmed by DNA sequencing. $5 \mu \mathrm{g}$ pTLN-hTRPV1 were linearized by Hpa I and purified. The in vitro transcription was carried out using $2 \mu \mathrm{g}$ linearized pTLN-hTRPV1 under the guideline of SP6 transcription kit (Ambion). TRPV1 cRNA was stored at $-20^{\circ} \mathrm{C}$ for the following experiments.

2.2. Cell Culture. Human mast cells HMC-1 (kindly provided by Dr. J. H. Butterfield, Mayo Clinic, USA) were cultured in IMDM (Gibco, Invitrogen, USA), supplemented with $2 \mathrm{mM}$ L-glutamine, $25 \mathrm{mM}$ HEPES, 10\% (v/v) fetal bovine serum (Gibco, Invitrogen), and 1\% penicillin and streptomycin (Gibco, Invitrogen, USA) in a 95\% humiditycontrolled incubator with $5 \% \mathrm{CO}_{2}$ at $37^{\circ} \mathrm{C}$.

2.3. Expression of TRPV1 Protein in Xenopus Oocytes. To investigate effects of laser irradiation on the TRPV1 protein, we used the Xenopus oocyte for heterologous expression and applied voltage-clamp techniques. Females of the clawed toad Xenopus laevis (Maosheng Bio-Technology Co., Shanghai, China) were anaesthetized with tricaine $\left(1 \mathrm{~g} / \mathrm{L} \mathrm{H}_{2} \mathrm{O}\right.$, MS222, Sandoz, Basel, Switzerland) or in ice water. Parts of the ovary were removed and treated with 0.3 units per $\mathrm{mL}$ liberase (Roche) for $3 \mathrm{~h}$ to remove enveloping tissue and to obtain isolated oocytes. For expression of TRPV1 protein, oocytes of stage V or VI [11] were selected and injected with $20 \mathrm{ng}$ cRNA (at $1 \mathrm{ng} / 1 \mathrm{~nL}$ ) two to three days before the experiments; uninjected oocytes served as controls. The cells were stored at $19^{\circ} \mathrm{C}$ in oocyte Ringer's-like solution (GORi, see Section 2.6). Experiments were performed at room temperature $\left(24-26^{\circ} \mathrm{C}\right)$.

2.4. Voltage-Clamp Experiments. We applied conventional two-electrode voltage clamp using Turbo TEC-03 with CellWorks software (NPI electronic, Tamm, Germany) to measure membrane currents. To determine steady-state currentvoltage dependencies (IV curves), membrane currents were averaged during the last $20 \mathrm{~ms}$ of $200 \mathrm{~ms}$, rectangular voltage pulses from -150 to $+30 \mathrm{mV}$ in $10 \mathrm{mV}$ increments; the pulses were applied from a holding potential of $-60 \mathrm{mV}$. To avoid changes at the reference bath electrode due to changes in $\mathrm{Cl}^{-}$ activity, the electrode was uncoupled from the bath via an ORi-filled channel.

2.5. Laser Stimulation. For laser stimulation of the oocytes continuous-wave (CW) lasers were used, for $406 \mathrm{~nm}$ the $\mathrm{CW}$
Laser 1051390/AF (COHERENT), for $532 \mathrm{~nm}$ the CW Laser SUWTECH LDC 1500 (Shanghai Uniwave Technology Co., Ldt), and for $657 \mathrm{~nm}$ the CW Laser SB2007047 (Shanghai University of Traditional Chinese Medicine). Fibre optics were used to guide the laser light close to the oocyte $(5 \mathrm{~mm})$ with output powers of $5 \mathrm{~mW}, 36 \mathrm{~mW}$, and 5-40 $\mathrm{mW}$-for the blue, red, and green laser light, respectively. The spot size at the position to the oocyte was $2 \mathrm{~mm}$ in diameter, oocytes had a diameter of $1-1.2 \mathrm{~mm}$. In several experiments using a one-millimetre thermoprobe, we confirmed that the applied laser light could not produce any significant change in temperature; at most an increase of 0.5 degrees was detectable after $30 \mathrm{~min}$ of irradiation. Taking into account that the oocyte was in addition continuously superfused with fresh solution of room temperature, effects of changing temperature can be excluded.

2.6. Solutions. Standard ORi (Oocyte Ringer's) solution contained (in mM) $90 \mathrm{NaCl}, 2 \mathrm{KCl}, 2 \mathrm{CaCl}_{2}$, and $5 \mathrm{MOPS}$ ( $\mathrm{pH} 7.4$, adjusted with Tris). For incubation of the oocytes, the ORi was supplemented with $70 \mathrm{mg} / \mathrm{L}$ Gentamycin (GORi). Stock solutions of capsaicin $(1 \mathrm{mM})$ were prepared in ethanol and of ruthenium red (RuR, $6 \mathrm{mM})$ in distilled water. The bath solution for HMC-1 cells contained (in mM) 150 $\mathrm{NaCl}, 5 \mathrm{KCl}, 2 \mathrm{CaCl}_{2}, 5 \mathrm{MgCl}_{2}, 4 \mathrm{D}$-sorbitol, and $10 \mathrm{HEPES}$ ( $\mathrm{pH} 7.4$ adjusted with $\mathrm{NaOH}$ ).

2.7. Data Analysis. For judging statistical significant effects of laser irradiation on current-voltage dependencies, $t$-tests were performed for at least 2 different potentials $(-100$ and $-50 \mathrm{mV}$ ). Mean values were considered as statistically different on the basis of $t, P<0.03$.

\section{Results}

3.1. Functional Expression of TRPV1 in the Oocytes. To demonstrate that TRPV1 was functionally expressed in the oocytes, several specific characteristics of TRPV1-mediated current were investigated.

TRPV1 is known to be activated by capsaicin [12, 13]. Oocytes injected with cRNA for TRPV1 responded to application of $500 \mathrm{nM}$ capsaicin with an increase in membrane current that completely disappeared after washout (Figure 1(a)). To correct for possible drift with time, capsaicin-induced current $I_{\text {cap }}$ was calculated according to

$$
I_{\text {cap }}=\frac{I 1_{\text {before }}-I 1_{\mathrm{after}}}{2}-I 2
$$

where $I 1$ is the current in the absence of capsaicin (before or after the application of the agonist) and $I 2$ the current in the presence. The difference current will be considered as current mediated by TRPV1 (Figure 1(b)); oocytes not injected with cRNA, never exhibited any capsaicin-sensitive current (Figure 1(b)). The current-voltage dependence is characterised by outward rectification. Oocytes not injected with cRNA exhibited no response to capsaicin. 


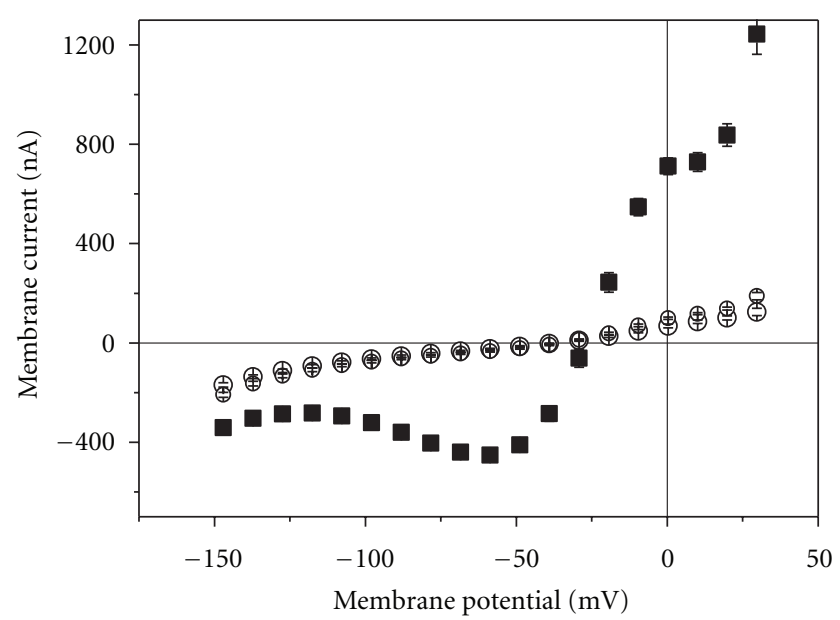

(a)

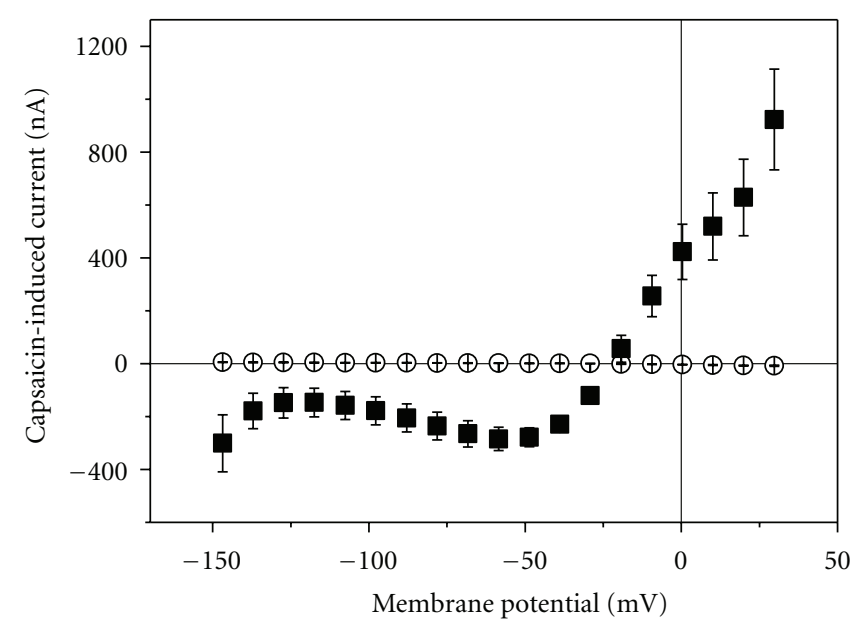

(b)

FIGURE 1: Effect of capsaicin application on current-voltage curves. (a) Membrane current in oocytes injected with TRPV1-cRNA. Large open circles before, filled squares during, and small open circles after application of $500 \mathrm{nM}$ capsaicin. (b) Capsaicine-induced current in uninjected oocytes (open circles) and cRNA-injected oocytes (filled squares). All data are averages from 8 experiments ( \pm SEM).

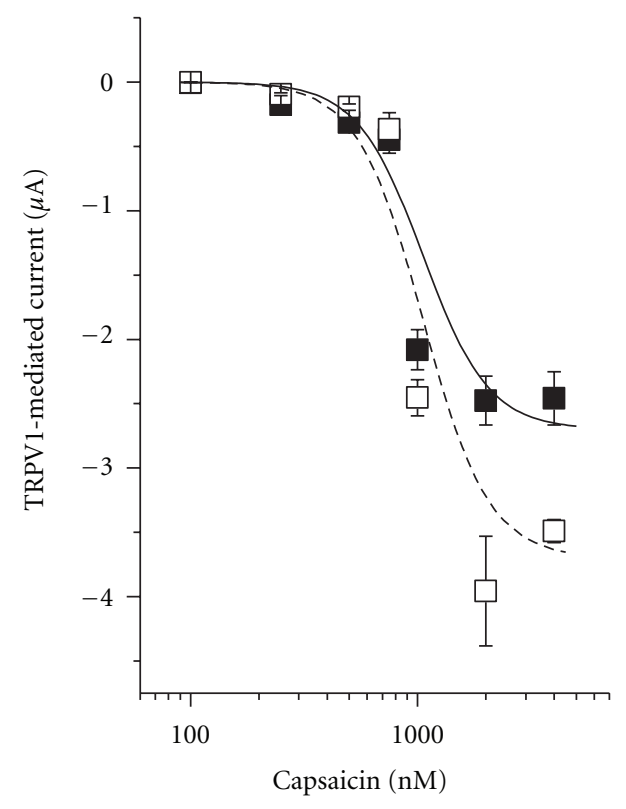

FIGURE 2: Dependence of TRPV1-mediated current on capsaicin concentration. Filled squares current at $-60 \mathrm{mV}$ and open squares at $-100 \mathrm{mV}$. Data are averages from 7 experiments $( \pm$ SEM). Lines represent approximations of the concentration dependencies with the same $K_{1 / 2}$ value of $1.06 \mu \mathrm{M}$.

The dependence of the TRPV1-mediated current showed strong dependence on capsaicin concentration (Figure 2) and can be approximated by

$$
I=I_{\max } \frac{[\text { capsaicin }]^{3}}{[\text { capsaicin }]^{3}+K_{1 / 2}^{3}},
$$

with an $K_{1 / 2}$ value of $1.06 \mu \mathrm{M}$ for -60 as well as $-100 \mathrm{mV}$.

Another characteristic of TRPV1 is its inhibition by ruthenium red (RuR) [14]. Figure 3 shows that $12 \mu \mathrm{M}$ RuR

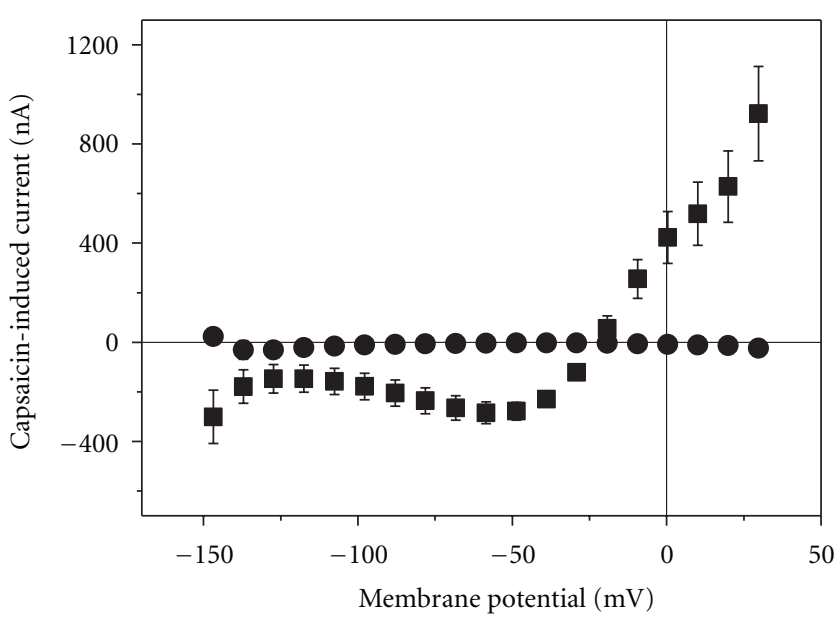

FIGURE 3: Inhibition of capsaicin-induced current by RuR. Filled square current-voltage dependence of current induced by $500 \mu \mathrm{M}$ capsaicin, filled circles in the simultaneous presence of $12 \mu \mathrm{M}$ RuR. Data are averages $( \pm$ SEM $)$ of 14 measurements in the absence and of 4 measurements in the presence of RuR.

completely blocked the capsaicin-induced current in the oocytes over the entire potential range. In control oocytes not injected with cRNA, no RuR-sensitive current component could be detected.

TRPV1 can also be activated by reduced $\mathrm{pH}$ [12]. Over the potential range of -40 to $-120 \mathrm{mV}$, reducing the extracellular $\mathrm{pH}$ hardly affected the membrane current in noninjected oocytes (Figure 4). In cRNA-injected oocytes, the change in $\mathrm{pH}$ from 7.4 to 6.2 activated a current (Figure 4) with similar voltage dependence as the capsaicininduced current (compare Figure 1(b)).

Physiologically TRPV1 functions as thermosensor responding to noxious temperatures exceeding $42^{\circ} \mathrm{C}$. If the temperature of the solution superfusing the oocyte was increased 


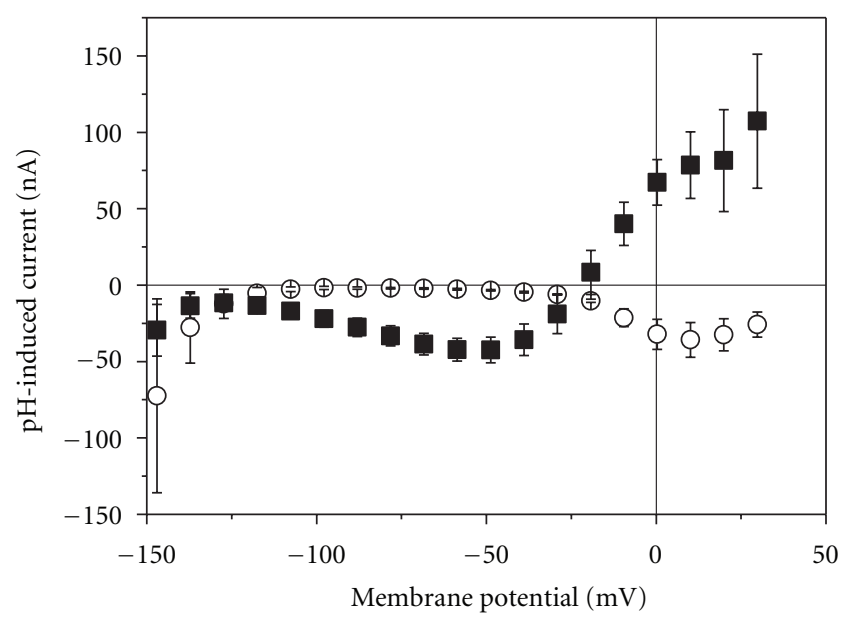

Figure 4: Effect of $\mathrm{pH}$ reduction on membrane current. Change current in response to a reduction of external $\mathrm{pH}$ from 7.4 to 6.2 in noninjected oocytes (open circles) and in cRNA-injected cells (filled squares). Data for injected cells are averages $( \pm$ SEM) of 7 measurement; for the control cells 2 measurements were performed.

from room temperature of $25^{\circ} \mathrm{C}$ to $42^{\circ} \mathrm{C}$, outward-rectifier current, typical for the capsaicin-induced current, was stimulated (Figure 5(a)). This increase in current was completely reversible when the temperature was returned to $25^{\circ} \mathrm{C}$. In the presence of RuR, no such current could be activated at $42^{\circ} \mathrm{C}$ (not illustrated). Also at $35^{\circ} \mathrm{C}$ only a tiny current component became apparent with voltage-dependence different from TRPV1-mediated current (Figure 5(b)).

In the following we investigated to which extent TRPV1 function can be modulated by laser light of three different wave lengths: red light of $637 \mathrm{~nm}$, blue light of $406 \mathrm{~nm}$, and green light of $532 \mathrm{~nm}$. In oocytes not expressing TRPV1 with none of the wavelengths, any current modulation was detectable, even at the highest output powers of $40 \mathrm{~mW}$.

3.2. Effect of Red Laser Light on TRPV1-Mediated Current. In medical low-level laser application, including laser acupuncture, red laser light is often applied. In the absence of capsaicin, no change in membrane current was detectable when red laser light $(637 \mathrm{~nm}, 5 \mathrm{~mW})$ was applied (not shown); even at $36 \mathrm{~mW}$ no sign of light-induced current was visible (for holding current at $-60 \mathrm{mV}$ see Figure 9). Also when TRPV1 was activated by $500 \mathrm{nM}$ capsaicin, a 2 min time period of irradiation could not be significantly modulated TRPV1-mediated current (Figure 6).

\subsection{Effect of Blue Laser Light on TRPV1-Mediated Current.} Recently, low-level blue laser light was introduced in laser acupuncture [9]. Therefore, we also tested for the effect of $406 \mathrm{~nm}$ on TRPV1-mediated current. Similarly to the red laser light, an output power of $5 \mathrm{~mW}$ could not affect membrane current (for holding current at $-60 \mathrm{mV}$ see Figure 9). Also when TRPV1 was activated by $500 \mathrm{nM}$ capsaicin, a $2 \mathrm{~min}$ time period of irradiation could not significantly modulate TRPV1-mediated current (Figure 7).
3.4. Effect of Green Laser Light on TRPV1-Mediated Current. In contrast to the red and blue laser light, green laser light $(532 \mathrm{~nm})$ activated in cRNA-injected oocytes even in the absence of capsaicin a current that increased with increasing output power (Figure 8(a)). Already at $5 \mathrm{~mW}$ a significant TRPV1-mediated current could be activated. The effect gradually increased with time reaching a maximum steady-state current within 2 min of irradiation (for $40 \mathrm{~mW}$ compare Figure 9). When the laser light was turned off, the current instantaneously dropped to the same level as before irradiation. At laser output power of $40 \mathrm{~mW}$, the typical outward rectification of TRPV1 channel was clearly apparent (Figure 8(b)).

3.5. Capsaicin Induces Degranulation in HMC-1. The experiments described above were done on the model system Xenopus oocyte. To support the idea that mast cell degranulation and activation of TRPV1 might be involved in acupuncture effects, we tested whether TRPV1 activation can induce mast cell degranulation. Figures $10(\mathrm{a})$ and $10(\mathrm{~b})$ illustrate that application of $1 \mu \mathrm{M}$ capsaicin (about $K_{1 / 2}$ value for TRPV1 activation, see Figure 2) indeed led to degranulation. Already at $500 \mathrm{nM}$ clear activation of TRPV1 was possible (Figure 1); after application of $500 \mathrm{nM}$ capsaicin for $5 \mathrm{~min} 62 \pm 8 \%$ of the cells had degranulated (Figure 10(c)).

\section{Discussion}

4.1. TRPV1 Is Functionally Expressed in the Oocytes. We have demonstrated that TRPV1 was functionally expressed in the oocytes by showing TRPV1-specific characteristics. Oocytes injected with the cRNA for TRPV1 exhibited capsaicininducible current (Figure 1) with a $K_{1 / 2}$ value of about $1 \mu \mathrm{M}$ (Figure 2) which is similar to the value reported by others $[12,13]$. Also the current-voltage dependence with outward-rectifier characteristic was reported for TRPV1mediated current $[14,15]$. The inhibition by RuR (Figure 3 ) and activation by reduced $\mathrm{pH}$ (Figure 4 ) of such outwardrectifying current are additional indications $[12,14]$ that TRPV1 was functionally expressed in the oocytes.

Within the TRPV family, TRPV1 is characterized also by temperature sensitivity with activation by temperature above $42^{\circ} \mathrm{C}[16,17]$. Increasing the temperature even to $35^{\circ} \mathrm{C}$ only slightly increased the membrane conductance (Figure 5(a)), but as soon as the temperature reached about $42^{\circ} \mathrm{C}$, current was induced with the outward-rectifying current-voltage dependence typical for TRPV1 (Figure 5(b)).

4.2. TRPV1 as a Candidate for Laser-Induced Degranulation. It was demonstrated previously that degranulation of mast cell is an essential initial step in acupuncture-induced pain relief [6]. In addition, it was demonstrated that various physical stimuli used in Chinese Medical Treatment can produce mast-cell degranulation [8]. These stimuli include mechanical stress, which is applied during the acupuncture needle manipulation [18-20] via the connective tissue to the mast cells, and high temperatures, which are applied during moxibustion to the acupuncture points [21]. Also 


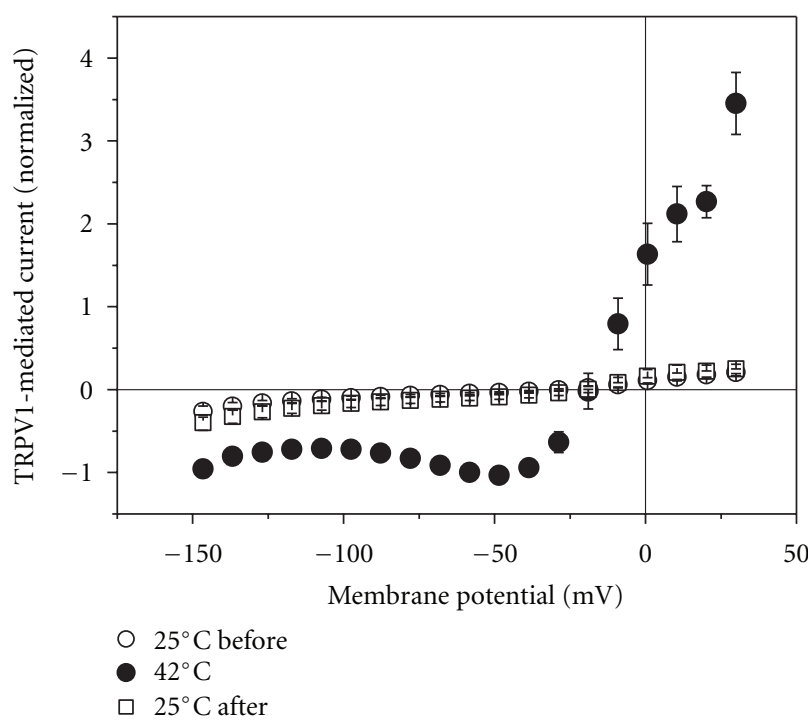

(a)

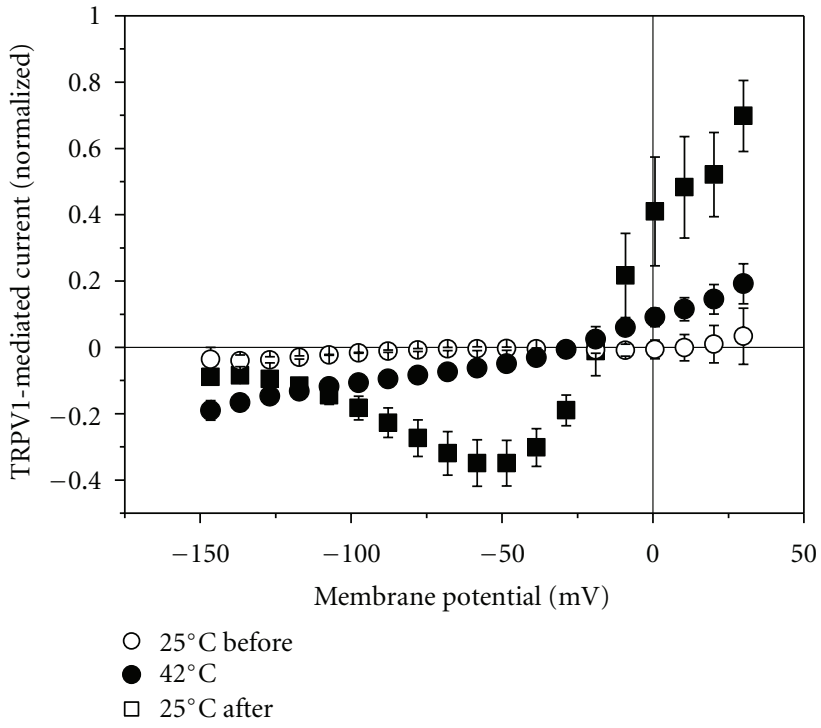

(b)

FIGURE 5: Effect of elevated temperature on membrane current of TRPV1-expressing oocytes. (a) Effect of $42^{\circ} \mathrm{C}$ (filled circles) in comparison to $25^{\circ} \mathrm{C}$ before and after the raise in temperature. (b) Effect of $35^{\circ} \mathrm{C}$ (filled circles) in comparison to $25^{\circ} \mathrm{C}$ before the raise in temperature and to the current induced by $500 \mathrm{nM}$ capsaicin (filled squares).

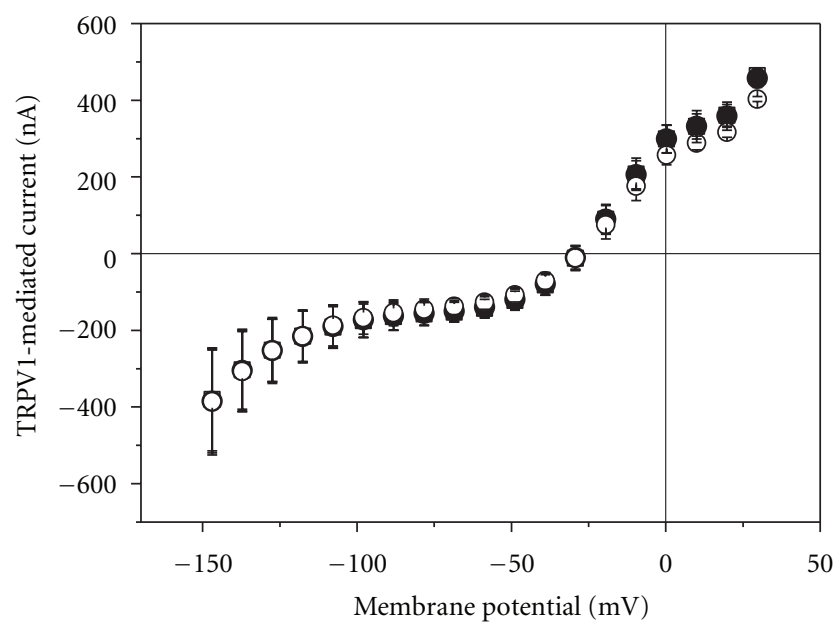

FIGURE 6: Effect of red laser light on current-voltage curve of TRPV1-mediated current. TRPV1 was activated by $500 \mathrm{nM}$ capsaicin. Open square were obtained before irradiation, filled circles at the end of a 2 min lasting irradiation period $(637 \mathrm{~nm}$, $36 \mathrm{~mW}$ ), and open circles $2 \mathrm{~min}$ after the laser light was turned off. Data represent averages from 3 oocytes $( \pm$ SEM).

red laser light in the 630 to $640 \mathrm{~nm}$ range is often applied to acupuncture points $[7,9,22]$. All three physical stimuli can elicit mast-cell degranulation that involves activation of TRPV2 ion channels [8].

Because of the optical window of tissue in the range of 600 to $1200 \mathrm{~nm}$, red and NIR light have a penetration depth of up to several mm [23] and have mainly been used to investigate photo biomodulation and stimulation including its application in laser acupuncture. Despite the

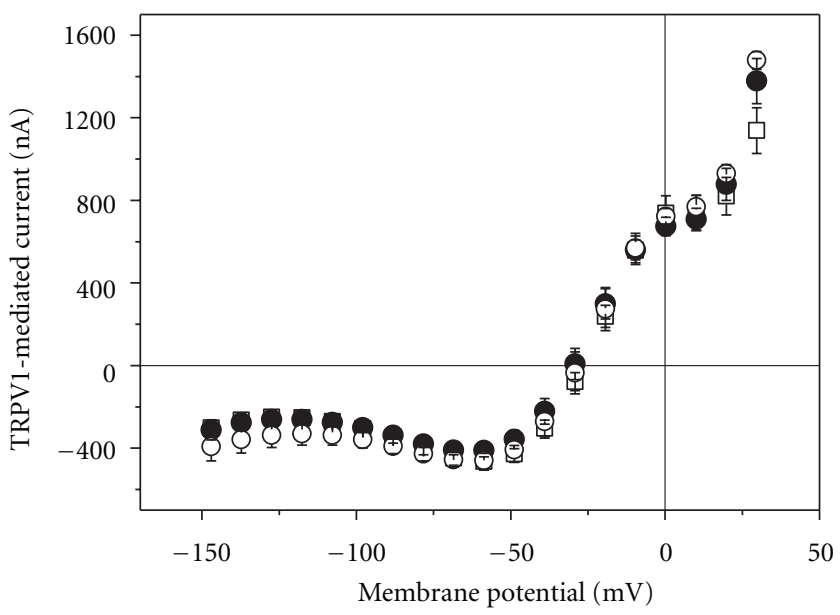

FIgURE 7: Effect of blue laser light on current-voltage curve of TRPV1-mediated current. TRPV1 was activated by $500 \mathrm{nM}$ capsaicin. Open square were obtained before irradiation, filled circles at the end of a 2-min lasting irradiation period $(406 \mathrm{~nm}$, $5 \mathrm{~mW}$ ), and open circles $2 \mathrm{~min}$ after the laser light was turned off. Data represent averages from 3 oocytes $( \pm$ SEM).

fact that blue light has a penetration depth in only the sub$\mathrm{mm}$ range, nevertheless, irradiation of acupuncture points by blue laser light was introduced into laser acupuncture recently [24]. Irradiation of mast cells with a $405 \mathrm{~nm}$ laser indeed leads to mast-cell degranulation that was attributed to an intracellular increase of $\mathrm{Ca}^{2+}$ by activation of TRPV4 [10]; TRPV4 is also expressed in mast cells $[8,10]$.

While red and blue laser light seem to stimulate activation of TRPV2 and TRPV4, respectively, these wave lengths were ineffective for TRPV1 (Figures 6 and 7). On the other 


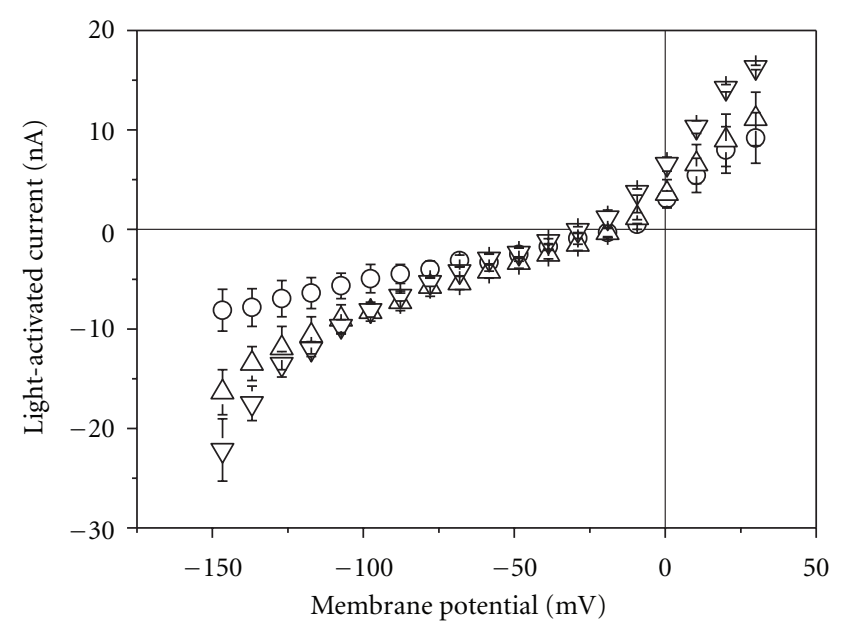

(a)

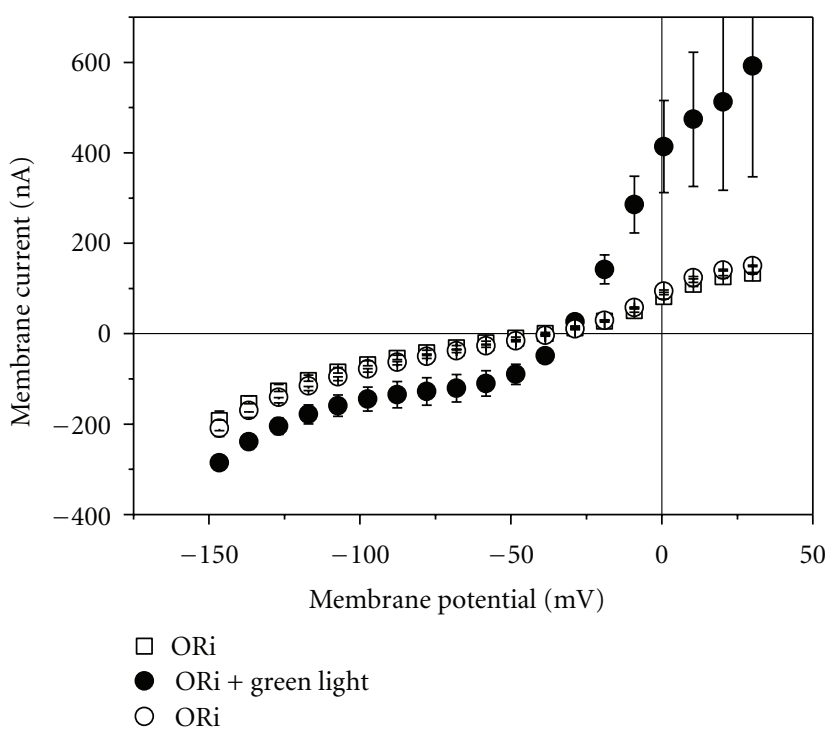

(b)

FIGURE 8: Effect of green laser light on current-voltage curve of TRPV1-mediated current. (a) Light-activated current in oocytes injected with cRNA for TRPV1; output power $5 \mathrm{~mW}$ (circles), $10 \mathrm{~mW}$ (triangle up), and $20 \mathrm{~mW}$ (triangles down). Data represent averages from 3 oocytes $( \pm$ SEM). (b) Membrane current before (open squares), during ( 2 min after light was turned on, filled circles), and after irradiation at $40 \mathrm{~mW}$. Data represent averages from 5 oocytes $( \pm$ SEM $)$.

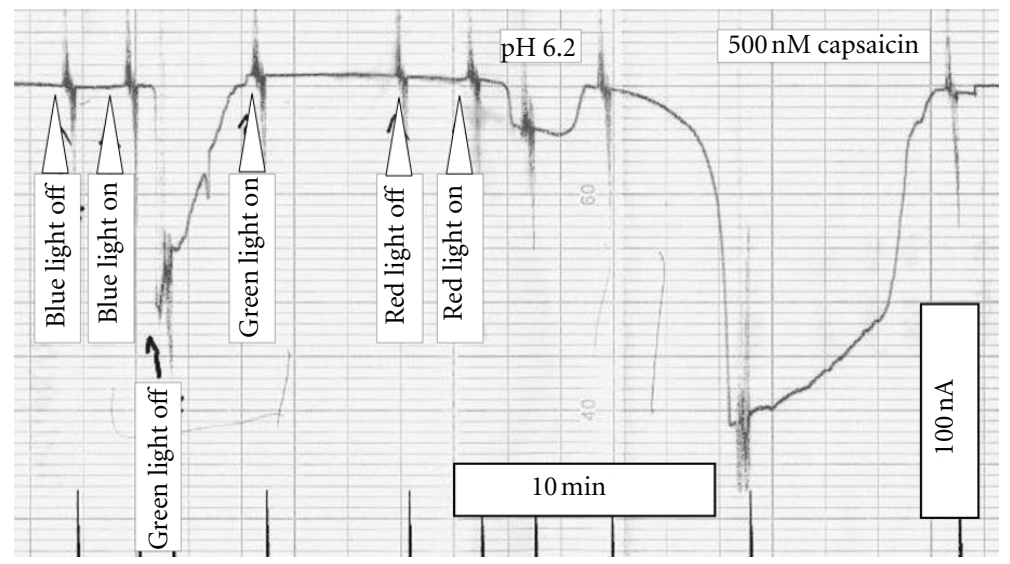

Figure 9: Pen recording of holding current at $-60 \mathrm{mV}$. Downward deflexions represent activation of inward current. Red light of $637 \mathrm{~nm}$ was applied at $36 \mathrm{~mW}$, green light of $532 \mathrm{~nm}$ at $40 \mathrm{~mW}$, and blue light of $406 \mathrm{~nm}$ at $5 \mathrm{~mW}$.

hand, for TRPV1 green laser light was a very effective stimulus (Figure 8). At $40 \mathrm{~mW}$, the light induced current amounted to about $80 \mathrm{nA}$ (at $-60 \mathrm{mV}$, see Figure 9), which is comparable to the $85 \mathrm{nA}$ of current that can be activated by $250 \mathrm{nM}$ capsaicin (Figure 2).

Photo biostimulation particularly in the red and NIR range have been attributed to absorption by cytochrome oxidase C. Interestingly, the stimulation of TRPV1 by the green light is dose-dependent, but after about 2 min a steady state is reached, and when turning off the light the TRPV1mediated current instantaneously vanishes. The physical basis for this is currently under investigation.

In our work, we focused on laser-light-induced mast-cell degranulation that might form an initial step in acupuncture induced analgesia. Detailed investigations have elucidated that irradiation of peripheral nerve with red or NIR laser light may also be of relevance for analgesic effects (for a detailed review see [25]). In particular, decreased conduction velocities have been reported in experiments on humans and animals with involvement of $\mathrm{A} \delta$ and $\mathrm{C}$ fibres. In our experiments, we could demonstrate the effectiveness of green laser light on TRPV1. Interestingly, TRPV1 is highly expressed in peripheral $\mathrm{A} \delta$ and $\mathrm{C}$ fibres [12], and hence, may also form in the nerve cells a target for green laser-light stimulation. It was reported that within acupuncture points, TRPV1 shows higher expression on $\mathrm{A} \delta$ and $\mathrm{C}$ fibres than in nonacupuncture points and this becomes even increased after electroacupuncture [26]. The authors conclude that the 


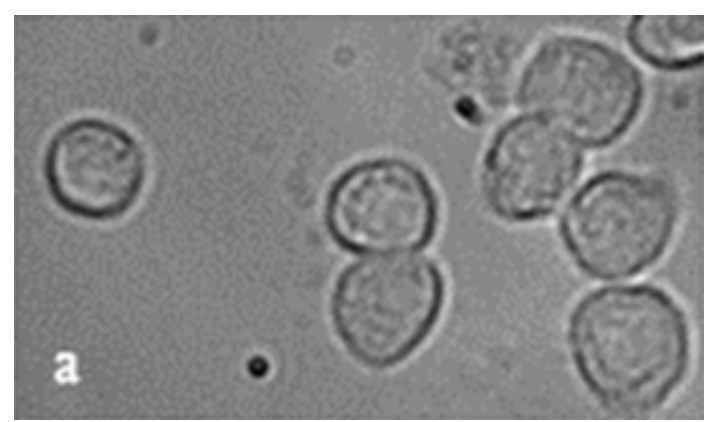

(a)

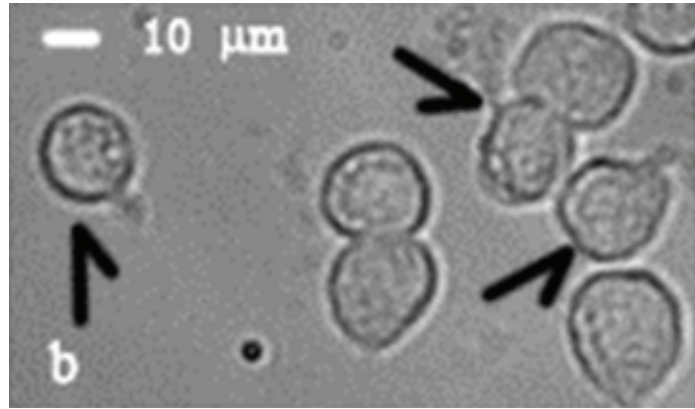

(b)

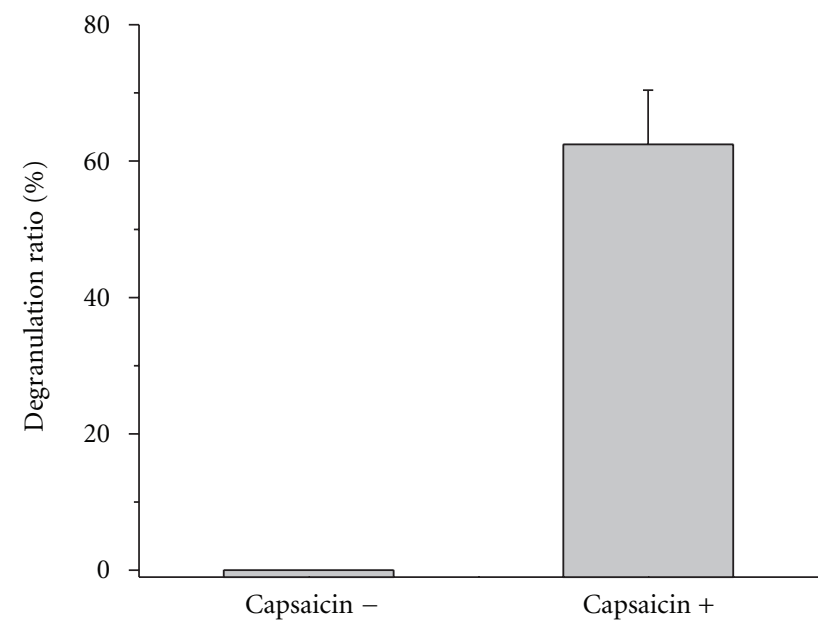

(c)

FIGURE 10: Degranulation of human mast cells induced by capsaicin. (a) Human mast cells HMC-1 incubated in bath solution in the absence of the TRPV1-specific agonist capsaicin. (b) HMC-1 cells having been superfused for 5 min with bath solution containing $1 \mu \mathrm{M}$ capsaicin. The arrows point to the degranulating cell. (c) Percentage of degranulated HMC-1 cells in the absence and presence of $500 \mathrm{nM}$ capsaicin (exposition time $10 \mathrm{~min}$, values present averages of 3 independent experiments $\pm \mathrm{SEM}$ ).

higher expression of TRPV1 and its upregulation in response to acupuncture may be involved in the transmission of the acupuncture signal.

In addition to the results in our model system Xenopus oocyte, we could show that activation of TRPV1 by the specific agonist capsaicin can indeed induce degranulation (Figure 10). We, therefore, like to suggest that in addition to red and blue laser light also green light with its intermediate penetration depth might be a useful tool in medical treatment.

\section{Abbreviations}

CW laser: Continuous-wave laser

ORi: $\quad$ Oocyte Ringer's

RuR: Ruthenium red

TRP: Transient receptor potential

TRPV1: Transient receptor potential valinoid-sensitive, isoform 1 .

\section{Acknowledgments}

The authors are very grateful to Dr. Bernd NILIUS for providing them the cDNA for TRPV1 and to Huiming
DU for his excellent technical assistance. The work was financially supported by the Science Foundation of Shanghai Municipal Commission of Science and Technology, the National Basic Research Program of China (973 Program, no. 2012CB518502), and the National Natural Science Foundation of China (no. 81102635).

\section{References}

[1] E. Mester, A. F. Mester, and A. Mester, "The biomedical effects of laser application," Lasers in Surgery and Medicine, vol. 5, no. 1, pp. 31-39, 1985.

[2] P. Whittaker, "Laser acupuncture: past, present, and future," Lasers in Medical Science, vol. 19, no. 2, pp. 69-80, 2004.

[3] T. Karu, "Primary and secondary mechanisms of action of visible to near-IR radiation on cells," Journal of Photochemistry and Photobiology B, vol. 49, no. 1, pp. 1-17, 1999.

[4] T. I. Karu, L. V. Pyatibrat, and N. I. Afanasyeva, "A novel mitochondrial signaling pathway activated by visible-to-near infrared radiation," Photochemistry and Photobiology, vol. 80, no. 2, pp. 366-372, 2004.

[5] G. Litscher, High-Tech Acupuncture \& Integrative Laser Medicine, Pabst Science Publishers, Lengerich, Germany, 2012, edited by G. Litscher, X. Y. Gao, L. Wang and B. Zhu. 
[6] D. Zhang, G. Ding, X. Shen et al., "Role of mast cells in acupuncture effect: a pilot study," Explore, vol. 4, no. 3, pp. 170-177, 2008.

[7] X. Y. Shen, L. Zhao, G. H. Ding et al., "Effect of combined laser acupuncture on knee osteoarthritis: a pilot study," Lasers in Medical Science, vol. 24, no. 2, pp. 129-136, 2009.

[8] D. Zhang, A. Spielmann, L. Wang, G. Ding, F. Huang, and Q. Gu, "Mast-cell degranulation induced by physicsl stimuli involves the activation of transient-receptor-potential channel TRPV2," Physiological Research, vol. 61, no. 1, pp. 113-124, 2012.

[9] G. Litscher, "Integrative laser medicine and high-tech acupunctre at the Medical University of Graz," Evidence-Based Complementary and Alternative Medicine, vol. 2012, Article ID 103109, 21 pages, 2012.

[10] W. Z. Yang, J. Y. Chen, J. T. Yu, and L. W. Zhou, "Effects of low power laser irradiation on intracellular calcium and histamine release in RBL-2H3 mast cells," Photochemistry and Photobiology, vol. 83, no. 4, pp. 979-984, 2007.

[11] J. N. Dumont, "Oogenesis in Xenopus laevis (Daudin). I. Stages of oocyte development in laboratory maintained animals," Journal of Morphology, vol. 136, no. 2, pp. 153-179, 1972.

[12] M. J. Caterina, M. A. Schumacher, M. Tominaga, T. A. Rosen, J. D. Levine, and D. Julius, "The capsaicin receptor: a heatactivated ion channel in the pain pathway," Nature, vol. 389, no. 6653, pp. 816-824, 1997.

[13] J. Vriens, G. Appendino, and B. Nilius, "Pharmacology of vanilloid transient receptor potential cation channels," Molecular Pharmacology, vol. 75, no. 6, pp. 1262-1279, 2009.

[14] C. Garcia-Martinez, C. Morenilla-Palao, R. Planells-Cases, J. M. Merino, and A. Ferrer-Montiel, "Identification of an aspartic residue in the P-loop of the vanilloid receptor that modulates pore properties," The Journal of Biological Chemistry, vol. 275, no. 42, pp. 32552-32558, 2000.

[15] M. Raisinghani, R. M. Pabbidi, and L. S. Premkumar, "Activation of transient receptor potential vanilloid 1 (TRPV1) by resiniferatoxin," Journal of Physiology, vol. 567, no. 3, pp. 771786, 2005.

[16] M. J. Caterina, A. Leffler, A. B. Malmberg et al., "Impaired nociception and pain sensation in mice lacking the capsaicin receptor," Science, vol. 288, no. 5464, pp. 306-313, 2000.

[17] R. Vennekens, G. Owsianik, and B. Nilius, "Vanilloid transient receptor potential cation channels: an overview," Current Pharmaceutical Design, vol. 14, no. 1, pp. 18-31, 2008.

[18] H. M. Langevin, D. L. Churchill, J. R. Fox, G. J. Badger, B. S. Garra, and M. H. Krag, "Biomechanical response to acupuncture needling in humans," Journal of Applied Physiology, vol. 91, no. 6, pp. 2471-2478, 2001.

[19] H. M. Langevin, "Connective tissue: a body-wide signaling network?" Medical Hypotheses, vol. 66, no. 6, pp. 1074-1077, 2006.

[20] H. M. Langevin, D. L. Churchill, J. Wu et al., "Evidence of connective tissue involvement in acupuncture," The FASEB Journal, vol. 16, no. 8, pp. 872-874, 2002.

[21] J. F. Zhang and Y. C. Wu, "Modern progress of mechanism of moxibustion therapy," Journal of Acupuncture and Tuina Science, vol. 4, no. 5, pp. 257-260, 2006.

[22] G. Litscher and D. Schikora, Laserneedle-Acupuncture: Science and Practice, Pabst Science Publishers, Lengerich, Germany, 2007, edited by G. Litscher and D. Schikora.

[23] R. R. Anderson and J. A. Parrish, "The optics of human skin," Journal of Investigative Dermatology, vol. 77, no. 1, pp. 13-19, 1981.
[24] X. Y. Gao, G. Litscher, K. Liu, and B. Zhu, "Sino-European transcontinental basic and clinical high-tech acupuncture studies-part 3: violet laser stimulation in anesthetized rats," Evidence-Based Complementary and Alternative Medicine, vol. 2012, Article ID 402590, 8 pages, 2012.

[25] R. Chow, P. Armati, E. L. Laakso, J. M. Bjordal, and G. D. Baxter, "Inhibitory effects of laser irradiation on peripheral mammalian nerves and relevance to analgesic effects: a systematic review," Photomedicine and Laser Surgery, vol. 29, no. 6, pp. 365-381, 2011.

[26] T. S. Abraham, M. L. Chen, and S. X. Ma, "TRPV1 expression in acupuncture points: response to electroacupuncture stimulation," Journal of Chemical Neuroanatomy, vol. 41, no. 3, pp. 129-136, 2011. 


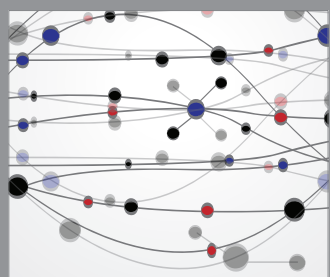

The Scientific World Journal
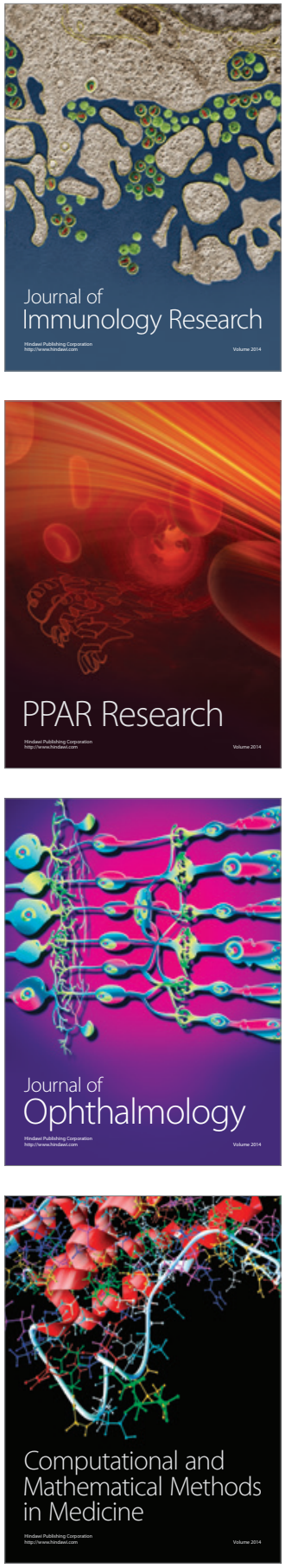

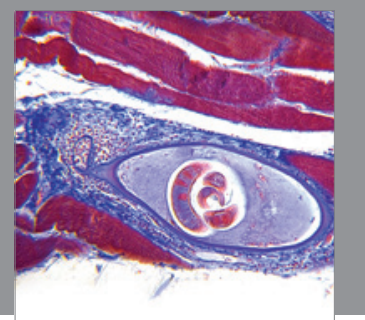

Gastroenterology

Research and Practice
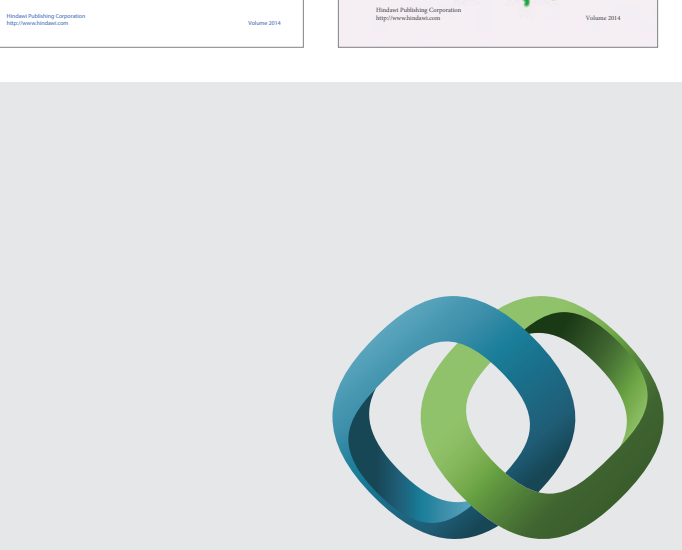

\section{Hindawi}

Submit your manuscripts at

http://www.hindawi.com
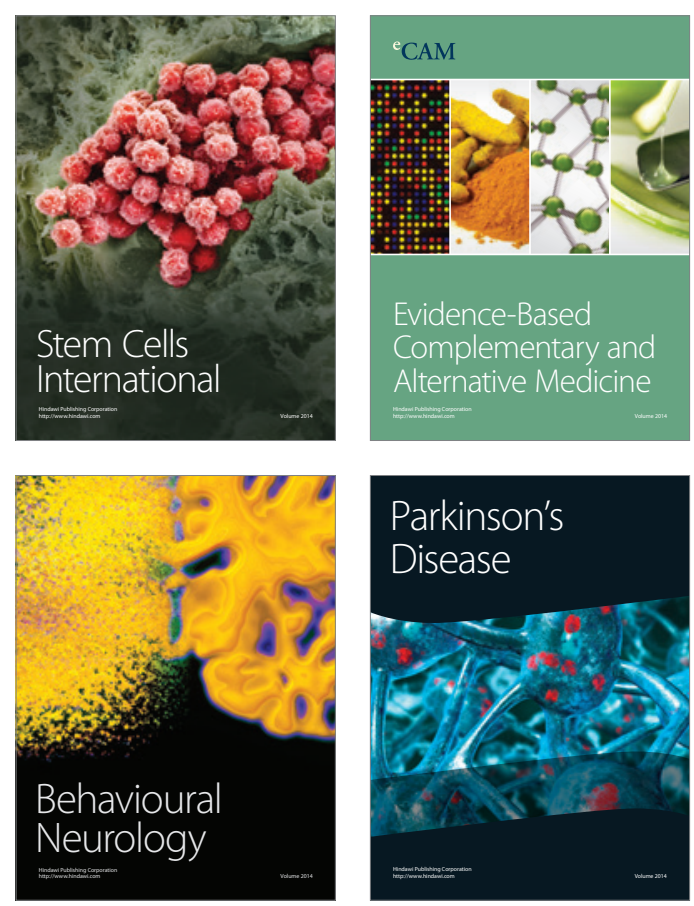

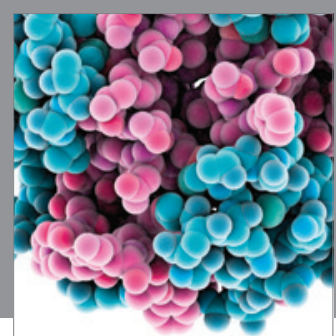

Journal of
Diabetes Research

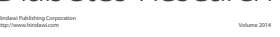

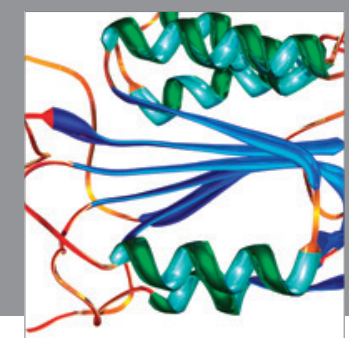

Disease Markers
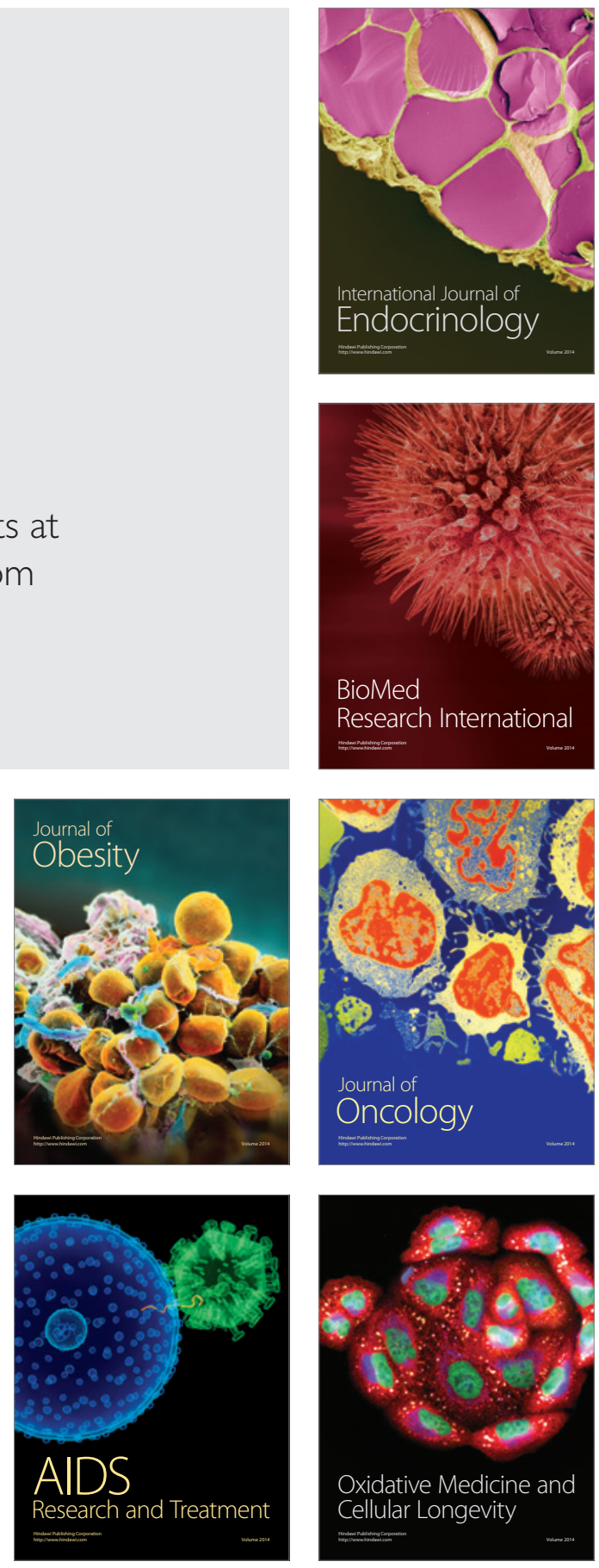